\title{
Anti-inflammatory effects of atorvastatin improve left ventricular function in experimental diabetic cardiomyopathy
}

\author{
S. Van Linthout • A. Riad • N. Dhayat • F. Spillmann • \\ J. Du • S. Dhayat • D. Westermann • \\ D. Hilfiker-Kleiner • M. Noutsias • U. Laufs • \\ H.-P. Schultheiss • C. Tschöpe
}

Received: 3 December 2006 / Accepted: 20 April 2007 / Published online: 23 June 2007

(C) Springer-Verlag 2007

\begin{abstract}
Aims/hypothesis Emerging evidence suggests that statins exert beneficial effects beyond those predicted by their cholesterollowering actions. We investigated whether atorvastatin influences the development of left ventricular (LV) dysfunction, independently of cholesterol-lowering, in an experimental model of type 1 diabetes mellitus cardiomyopathy.

Methods Streptozotocin-induced diabetic rats were treated with atorvastatin $(50 \mathrm{mg} / \mathrm{kg}$ daily, orally) or with vehicle for 6 weeks. LV function was analysed using tip-catheter measurements. Cardiac stainings of TNF- $\alpha$, IL- $1 \beta$, intercellular adhesion molecule-1, vascular cellular adhesion molecule-1, CD11a/lymphocyte-associated antigen-1, CD11b/ macrophage antigen alpha, CD18/ß2-integrin, ED1/CD68, collagen I and III, and Sirius Red were assessed by digital image analysis. Ras-related C3 botulinum toxin substrate (RAC1) and ras homologue gene family, member A (RHOA)
\end{abstract}

S. Van Linthout $\cdot$ A. Riad $\cdot$ N. Dhayat $\cdot$ F. Spillmann $\cdot$ J. Du $\cdot$

S. Dhayat $\cdot$ D. Westermann $\cdot$ M. Noutsias $\cdot$ H.-P. Schultheiss

C. Tschöpe $(\square)$

Department of Cardiology and Pneumology,

Campus Benjamin Franklin, Charité-University Medicine Berlin,

Hindenburgdamm 30,

12200 Berlin, Germany

e-mail: ctschoepe@yahoo.com

D. Hilfiker-Kleiner

Department of Cardiology, Medical School Hannover, Hannover, Germany

U. Laufs

Department of Cardiology, University of Saarland,

Homburg/Saar, Germany activities were determined by RAC1 glutathione- $S$-transferasep21-activated kinase and rhotekin pull-down assays, respectively. Cardiac lipid peroxides were measured by a colorimetric assay. The phosphorylation state of p38 mitogen-activated protein kinase (MAPK) and endothelial nitric oxide synthase (eNOS) protein production were analysed by western blot.

Results Diabetes was associated with induced cardiac stainings of TNF- $\alpha$, IL-1 $\beta$, cellular adhesion molecules, increased leucocyte infiltration, macrophage residence and cardiac collagen content. In contrast, atorvastatin reduced both intramyocardial inflammation and myocardial fibrosis, resulting in improved LV function. This effect was paralleled with a normalisation of diabetes-induced RAC1 and RHOA activity, in the absence of LDL-cholesterol lowering. In addition, atorvastatin decreased diabetesinduced cardiac lipid peroxide levels and p38 MAPK phosphorylation by 1.3 -fold $(p<0.05)$ and 3.2-fold $(p<0.0005)$, respectively, and normalised the reduced eNOS production caused by diabetes.

Conclusions/interpretation These data indicate that atorvastatin, independently of its LDL-cholesterol-lowering capacity, reduces intramyocardial inflammation and myocardial fibrosis, resulting in improved LV function in an experimental model of diabetic cardiomyopathy.

Keywords Atorvastatin - Diabetes mellitus .

Diabetic cardiomyopathy · Fibrosis · Inflammation .

LDL-cholesterol - Left ventricular function · p38 MAPK .

$\mathrm{RAC} 1 \cdot \mathrm{RHOA}$

\section{Abbreviations}

CAM cellular adhesion molecules

$\mathrm{d} P / \mathrm{d} t_{\text {max. }} \quad$ maximal rate of left ventricular pressure rise 


$\begin{array}{ll}\mathrm{d} P / \mathrm{d} t_{\min .} & \begin{array}{l}\text { minimal rate of left ventricular pressure fall } \\ \text { endothelial nitric oxide synthase }\end{array} \\ \text { eNOS } & \text { 3-hydroxy-3-methylglutaryl coenzyme A } \\ \text { ICAM-1 } & \begin{array}{l}\text { intercellular adhesion molecule-1 } \\ \text { LFA-1 }\end{array} \\ \text { lymphocyte function associated antigen 1 } \\ \text { lV } & \text { left ventricular } \\ \text { MAC1 } & \text { macrophage antigen alpha } \\ \text { MAPK } & \text { mitogen-activated protein kinase } \\ \text { RAC1 } & \text { ras-related C3 botulinum toxin substrate } \\ \text { RHOA } & \text { ras homologue gene family, member A } \\ \text { SD } & \text { Sprague-Dawley } \\ \text { VCAM-1 } & \text { vascular cellular adhesion molecule-1 }\end{array}$

\section{Introduction}

Diabetes mellitus is an independent risk factor for left ventricular (LV) dysfunction [1] in type 1 and 2 diabetes mellitus and is associated with a specific diabetic cardiomyopathy [2]. Several mechanisms, of which the relative significance is still not known, are involved in the pathogenesis of diabetic cardiomyopathy, including myocardial fibrosis, interstitial inflammation and endothelial dysfunction [2-4].

Diabetes-associated hyperglycaemia and dyslipidaemia [5] are associated with pro-inflammatory effects, thereby affecting the endothelium. Hyperglycaemia induces the appearance of cellular adhesion molecules (CAMs), leading to increased adherence of monocytes [3]; it also alters the permeability of the endothelial basement membrane, leading to accelerated lipid deposition and macrophage recruitment [6]. In addition, hyperglycaemia-associated oxidative stress enhances the oxidative modification of LDLs [7], which themselves induce production of CAMs and increase monocyte adhesion [8].

By blocking the conversion of 3-hydroxy-3-methylglutaryl coenzyme A (HMG CoA) to mevalonate, HMG CoA reductase inhibitors (statins) are potent inhibitors of cholesterol and isoprenoid biosynthesis. Recent experimental and clinical evidence indicates that the effects of statins may extend beyond their ability to reduce serum cholesterol level $[9,10]$. Cholesterol-independent or pleiotropic effects of statins include improved endothelial function, and reduction of oxidative stress and vascular inflammation. Many of these pleiotropic effects are mediated by antagonism of isoprenoid-mediated activation of small GTPbinding proteins, such as RHO family members, including ras-related $\mathrm{C} 3$ botulinum toxin substrate (RAC1) and ras homologue gene family, member A (RHOA) [11], activation of the latter being associated with decreased endothelial nitric oxide synthase (eNOS) expression and activity
$[12,13]$. The cardioprotective effects of statins, independent of their hypolipidaemic effects, have already been demonstrated in normocholesterolaemic animal models of hypertrophy [14] and myocardial infarction [15]. However, their pleiotropic effects have not been investigated before in an animal model associated with increased LDL-cholesterol.

The aim of this study was to investigate whether atorvastatin reduces intramyocardial inflammation and myocardial fibrosis, independently of LDL-cholesterol-lowering, thereby inhibiting the development of LV dysfunction in an experimental model of diabetic cardiomyopathy that is associated with increased LDL-cholesterol.

\section{Methods}

Animals and study design Male Sprague-Dawley (SD) rats (8 weeks old; 300-330 g; Charles River WIGA, Sulzfeld, Germany) were maintained on a $12 \mathrm{~h}$ light-dark cycle, with free access to standard chow. Diabetes mellitus was induced by a single injection of streptozotocin (STZ; $70 \mathrm{mg} / \mathrm{kg}$; i.p.) prepared in $0.1 \mathrm{~mol} / 1$ sodium citrate buffer, $\mathrm{pH} 4.5$ (Sigma, Munich, Germany), as described in detail elsewhere [16]. Only rats with blood glucose levels $\geq 16.7 \mathrm{mmol} / 15$ days after STZ injection were used in the study. One diabetic group was treated with atorvastatin (STZ + atorvastatin) $(50 \mathrm{mg} / \mathrm{kg}$ daily, orally) for 6 weeks, starting 5 days after STZ injection. In parallel, a non-diabetic group (SD + atorvastatin) was treated with atorvastatin to analyse basal parameters, verifying potential side effects of atorvastatin. Non-diabetic (SD) and diabetic (STZ) control rats were treated with vehicle. The dose of atorvastatin, chosen to not affect LDL-cholesterol in STZ rats, was determined in a preliminary experiment where 50 or $100 \mathrm{mg} / \mathrm{kg}$ per day of atorvastatin were administered orally. The experiment revealed that in contrast to $100 \mathrm{mg} / \mathrm{kg}$ daily, which reduced LDL-cholesterol by $34 \%$, the lower dose did not significantly decrease LDL-cholesterol levels in STZ-induced diabetic rats.

At the end of the experimental protocol, after haemodynamic characterisation, the hearts were excised and transverse sections (about $0.2 \mathrm{~cm}$ thick) of the hearts were rapidly frozen in liquid nitrogen and stored at $-80^{\circ} \mathrm{C}$ for immunohistology, western blot, real-time PCR, pull-down and lipid peroxide assays. A further section was paraffin embedded for Sirius Red staining. In addition, blood from the non-fasting animals was withdrawn from the aorta for determination of serum triacylglycerol, total cholesterol, and LDL- and HDL-cholesterol.

All experimental procedures were performed according to the Guide for the Care and Use of Laboratory Animals 
published by the US National Institutes of Health (NIH Publication No. 85-23, revised 1996).

Haemodynamic characterisation Heart rate (beats per min), the maximal rate of $\mathrm{LV}$ pressure rise $\left(\mathrm{d} P / \mathrm{d} t_{\text {max }}, ; \mathrm{mmHg} / \mathrm{s}\right)$ and the minimal rate of $\mathrm{LV}$ pressure fall $\left(\mathrm{d} P / \mathrm{d} t_{\min } ; \mathrm{mmHg} / \mathrm{s}\right)$ were recorded via a Millar-tip catheter $(2 \mathrm{~F})$ system (Millar Instruments Inc., Houston, TX, USA) in anaesthetised (ketamine [50 mg/kg; i.p.], $2 \%$ xylasine [5 $\mathrm{mg} / \mathrm{kg}$; i.p.]), ventilated ( $n=8$ per group), open-chest animals as described in detail elsewhere [17].

Sirius Red staining Total collagen content of the Sirius Red (Polyscience, Warrington, PA, USA) stained sections was measured under circularly polarised light according to previously published methods [18] and quantified by digital image analysis as described in detail elsewhere [19]. Perivascular fibrosis was not measured. All available fields (>30 fields) were measured, including the septum, the right and the left ventricle. Data, represented as area fraction, are depicted as medians and interquartile ranges (25th-75th percentile in parentheses).

Immunohistology Immunohistological stainings were carried out with mouse anti-rat (intercellular adhesion molecule-1 [ICAM-1], CD11a/lymphocyte function associated antigen 1 [LFA-1], CD11b/macrophage antigen alpha [MAC1], CD18/ß2-integrin and ED1/CD68; Serotec, Munich, Germany; and vascular cellular adhesion molecule-1 [VCAM-1], Hiss Diagnostics, Freiburg, Germany), goat anti-rat (TNF- $\alpha$ and IL-1 $\beta$; R and D Systems, Wiesbaden, Germany) or rabbit anti-collagen subtype I and III antibodies, and quantified by digital image analysis as described previously [3, 19]. All available fields $(>30$ fields) were measured, including the septum, and the right and the left ventricle. CAM data, represented as area fraction, and other immunohistological data, represented as infiltrate $/ \mathrm{mm}^{2}$, are depicted as medians and interquartile ranges (25th-75th percentile in parentheses).

Real-time RT-PCR Quantitative real-time RT-PCR (ABI PRISM 7900 HT Sequence Detection System software version 2.2.2; Applied Biosystems, Foster City, CA, USA) was performed to quantify rat LV Icam-1 and Vcam-1 cDNA levels. The levels of Icam-1 and Vcam-1 cDNA were normalised to the levels of the ribosomal protein L32 (L32; also known as Rprl32) housekeeping gene cDNA. As DNA standards, the conventional PCR products of rat ICAM-1, VCAM-1 and L32 with the use of the primers designed for the real-time PCR, were cloned into pGEM-T Easy vector (Promega Corporation, Madison, WI, USA). The sequences of the primer sets used in this study were as follows: for Icam-1 5'-GTCTCATGCCCGTGAAATTATG-3' and 5'-CATTTTCT
CCCAGGCATTCTCT-3', for Vcam-1 5'-GGAGGTCTACT CATTCCCTGAAGA- $3^{\prime}$ and $5^{\prime}$-ACCGTGCAGTTGACAG TGACA-3' and for L32 5'-AACCGAAAAGCCATCGT AGAAA-3' and REV: 5'-CCTGGCGTTGGGATTGG-3'.

RAC1 glutathione-S-transferase-p21-activated kinase and rhotekin pull-down assays RAC1 and RHOA activities were determined by pull down assays. A glutathione- $S$ transferase (GST)-p21-activated kinase (PAK)-CD (PAKCRIB domain) fusion protein, containing the $R A C l$ binding region from human p21 (CDKN1A)-activated kinase 1 (PAK1B) was used to determine RAC1 activity as described [20]. GST-C21 containing the RHO binding region from the RHO effector protein rhotekin was used to measure RHOA activity [21]. The constructs were a kind gift of R. C. Roovers and J. G. Collard (The Netherlands Cancer Institute, Amsterdam, The Netherlands).

Western blot LV samples were homogenised in lysis buffer containing proteinase inhibitors. An equal amount of protein was loaded into a $10 \%$ SDS-polyacrylamide gel. Total p38 mitogen-activated protein kinase (MAPK) (Cell Signaling Technology, Danvers, MA, USA), phosporylated p38 MAPK (Cell Signaling Technology) and total eNOS (BD Biosciences, San Diego, CA, USA) were detected with each specific antibody. The blots were visualised with electrochemiluminescence (Amersham Bioscience, Bucks, UK). Quantitative analysis of the intensity of the bands was performed with NIH Image 1.63 Software (http://rsb.info. nih.gov/nih-image/download.html). Equal protein loading was confirmed by gel staining with SimplyBlue Safestain (Invitrogen, Carlsbad, CA, USA) after SDS-PAGE.

Oxidative stress assay The presence of malondialdehyde, a biomarker of lipid peroxidation, in septum was examined by the use of a kit (Bioxytech LPO-586; OxisResearch, Foster City, CA, USA), according to the manufacturer's protocol. In brief, proteins were extracted from septa in icecold PBS (20 mmol/l, pH 7.4). To prevent sample oxidation during homogenisation, $10 \mu \mathrm{l}$ of $0.5 \mathrm{~mol} / 1$ butylated hydroxytoluene in acetonitrile was added per $\mathrm{ml}$ of tissue homogenate. Next, the homogenate was centrifuged for $10 \mathrm{~min}$ at $3,000 \mathrm{~g}$ and at $4^{\circ} \mathrm{C}$. The clear supernatant fraction was used for the colorimetric assay.

Statistical analysis Statistical analysis was performed using JMP Statistical Discovery Software, version 4.05 (SAS Institute, Cary, NC, USA). Non-parametric tests were used for haemodynamic and collagen data analysis, since normal distribution was excluded for all parameters by conducting the Shapiro-Wilk W test $(p<0.05)$. The statistically significant difference for multiple comparisons of all pairs of ordinal and continuous data was calculated by the 
Table 1 Metabolic parameters

\begin{tabular}{lllll}
\hline & SD & SD + atorvastatin & STZ & STZ + atorvastatin \\
\hline Body weight (g) & $480 \pm 45^{*}$ & $452 \pm 5.5^{*}$ & $219 \pm 35$ & $231 \pm 28$ \\
Blood glucose (mmol/l) & $10.5 \pm 0.42^{*}$ & $10 \pm 0.44^{*}$ & $44 \pm 1.8$ & $44 \pm 2.8$ \\
Total cholesterol (mmol/1) & $1.3 \pm 0.092^{* *}$ & $1.5 \pm 0.16^{* *}$ & $3.1 \pm 0.49$ & $2.8 \pm 0.36$ \\
HDL-cholesterol (mmol/l) & $0.90 \pm 0.067^{* * *}$ & $0.72 \pm 0.16^{* * *}$ & $1.2 \pm 0.11$ & $1.3 \pm 0.097$ \\
LDL-cholesterol (mmol/l) & $0.24 \pm 0.015^{* *}$ & $0.26 \pm 0.038^{* *}$ & $0.82 \pm 0.11$ & $0.74 \pm 0.12$ \\
Triacylglycerol (mmol/l) & $1.0 \pm 0.20^{* *}$ & $0.84 \pm 0.16^{* *}$ & $6.9 \pm 1.3$ & $5.6 \pm 1.1$ \\
\hline
\end{tabular}

Data are mean $\pm \mathrm{SE}$

${ }^{*} p<0.0001$ vs STZ and STZ + atorvastatin

$* * p<0.01$ vs STZ and STZ + atorvastatin

$* * * p<0.05$ vs STZ and STZ + atorvastatin

Tukey-Kramer post hoc analysis. Paired and unpaired Student's $t$ tests and ANOVA for multiple comparisons were employed for RAC1 and RHOA, real-time PCR, western blot and oxidative stress data analysis. Differences were considered significant at $p<0.05$ in all tests performed.

\section{Results}

Metabolic parameters Throughout the 6 week study period, atorvastatin-treated and control diabetic rats displayed severe hyperglycaemia, polydipsia, polyphagia and polyuria. This was associated with loss in body weight, resulting in a significantly higher heart:body weight ratio (\%) compared with the non-diabetic groups (SD: 0.28 \pm 0.008 ; SD + atorvastatin: $0.26 \pm 0.007$; STZ: $0.38 \pm 0.007$; STZ + atorvastatin: $0.34 \pm 0.004$ ) (Table 1). Diabetic animals had higher plasma levels of total cholesterol, LDL-cholesterol, HDL-cholesterol and triacylglycerol compared with nondiabetic animals. Atorvastatin treatment did not affect lipid levels, either in diabetic or in non-diabetic rats (Table 1).

Haemodynamics STZ-induced diabetic animals showed a significant reduction of $\mathrm{d} P / \mathrm{d} t_{\max }$ and $\mathrm{d} P / \mathrm{d} t_{\min }$ compared with non-diabetic ones. Importantly, LV function in atorvastatin-treated STZ rats was significantly improved compared with STZ controls and showed no significant difference compared with non-diabetic controls as assessed by Tukey-Kramer post-hoc analysis (Table 2). Atorvastatin treatment in non-diabetic animals did not affect LV function, indicating no potential side effects of atorvastatin. Due to the anaesthesia all animals had bradycardia. However, no significant differences in heart rate were found among the different animal groups.

Effect of atorvastatin treatment on fibrosis in the diabetic heart Since changes in the collagen composition, and particularly in collagen type I and III, influence the passive mechanical properties of the myocardium and thus are important for cardiac haemodynamics [22], we analysed total collagen content and collagen type I and III in the rat hearts. In agreement with the improved contractility in STZ-rats after atorvastatin treatment, atorvastatin blunted diabetes-induced interstitial fibrosis (Fig. 1a-c,j). Collagen types I and III content in the hearts of atorvastatin-treated STZ rats were 9.6-fold and 2.9-fold lower, respectively, compared with STZ rat hearts, leading to a twofold $(p<0.05)$ decrease in collagen type I:type III ratio (SD: $0.21 \pm 0.03$, STZ: $0.30 \pm$ 0.038, STZ + atorvastatin: 0.15 \pm 0.026 ) (Fig. 1d-j).

Effect of atorvastatin treatment on inflammation in the diabetic heart Given the importance of inflammation in the induction of fibrosis, we analysed CAM levels, leucocyte infiltration, macrophage residence and cytokine levels in the rat hearts. Quantification by digital image analysis demonstrated a significant increase in ICAM-1 and VCAM-1 stainings in STZ-diabetic rats compared with non-diabetic controls (Fig. 2a,b,d,e). Atorvastatin treatment in STZ rats resulted in a 16-fold $(p<0.05)$ and 36-fold $(p<0.05)$

Table 2 Haemodynamic parameters

\begin{tabular}{llll}
\hline & SD & SD + atorvastatin & STZ \\
\hline Heart rate $(\mathrm{bpm})$ & $191 \pm 32$ & $184 \pm 26$ & $180 \pm 36$ \\
$\mathrm{LV} \mathrm{dP} / \mathrm{d} t_{\max }(\mathrm{mmHg} / \mathrm{s})$ & $5,667 \pm 535^{*}$ & $5,650 \pm 595^{*}$ & $2,697 \pm 282$ \\
$\mathrm{LV} \mathrm{dP} / \mathrm{d} t_{\min }(\mathrm{mmHg} / \mathrm{s})$ & $-4,667 \pm 419^{*}$ & $-3,957 \pm 348^{*}$ & $-2,218 \pm 209$ \\
\hline
\end{tabular}

Data are mean \pm SEM

bpm beats per min

$* p<0.05$ vs STZ 

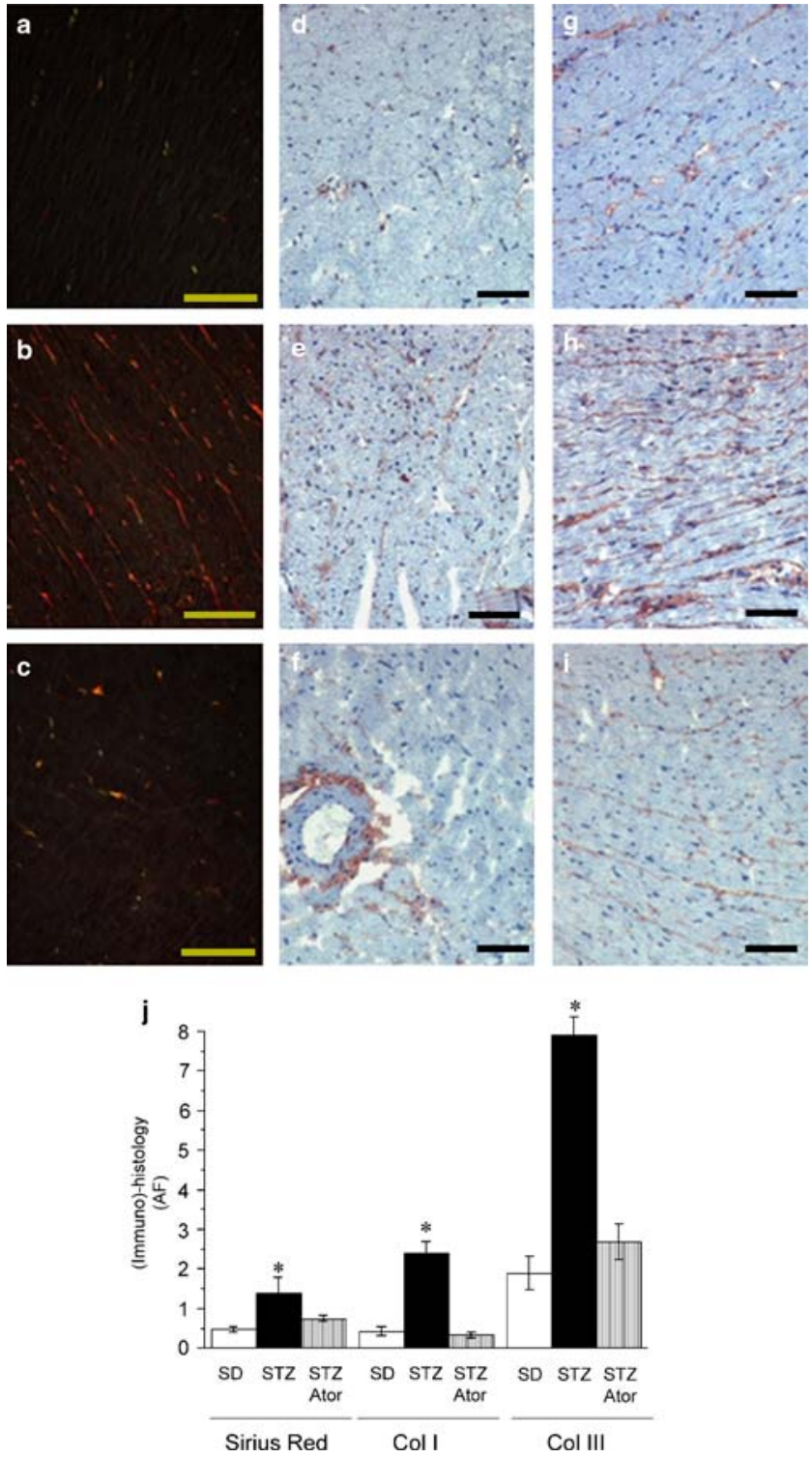

Fig. 1 Effect of atorvastatin treatment on cardiac levels of total collagen, collagen I and III in STZ-diabetic rat hearts. Sirius Red staining (original magnification: $\times 200$ ) in $($ a) a non-diabetic SD rat heart, (b) an STZ-diabetic rat heart and (c) an atorvastatin-treated (Ator) STZ-diabetic rat heart. Immunohistochemistry of collagen type I (original magnification: $\times 100)(\mathbf{d}-\mathbf{f})$ in a non-diabetic SD rat heart, an STZ-diabetic rat heart and an atorvastatin-treated STZ-diabetic rat heart respectively. g-i Immunohistochemistry of collagen type III (original magnification: $\times 100$ ) in a non-diabetic SD rat heart $(\mathbf{g})$ and an STZ-diabetic rat heart (h), with (i) reduced collagen type III expression in an atorvastatin-treated STZ-diabetic rat heart. Bar (a-c): $200 \mu \mathrm{m} ;(\mathbf{d}-\mathbf{i}): 100 \mu \mathrm{m}$. j Bar graph representing quantification of Sirius Red, collagen type I ( $\mathrm{Col} I)$ and collagen type III ( $\mathrm{Col}$ III) by (immuno)-histology. $A F$, area fraction. ${ }^{*} p<0.05$ vs $\mathrm{SD}$ and $\mathrm{STZ}+$ atorvastatin

reduction in ICAM-1 and VCAM-1 stainings, respectively, which was further confirmed by a significant reduction of LV Icam-1 and Vcam-1 mRNA expression (Fig. 2g). In agreement with the reduced CAM abundance, atorvastatin
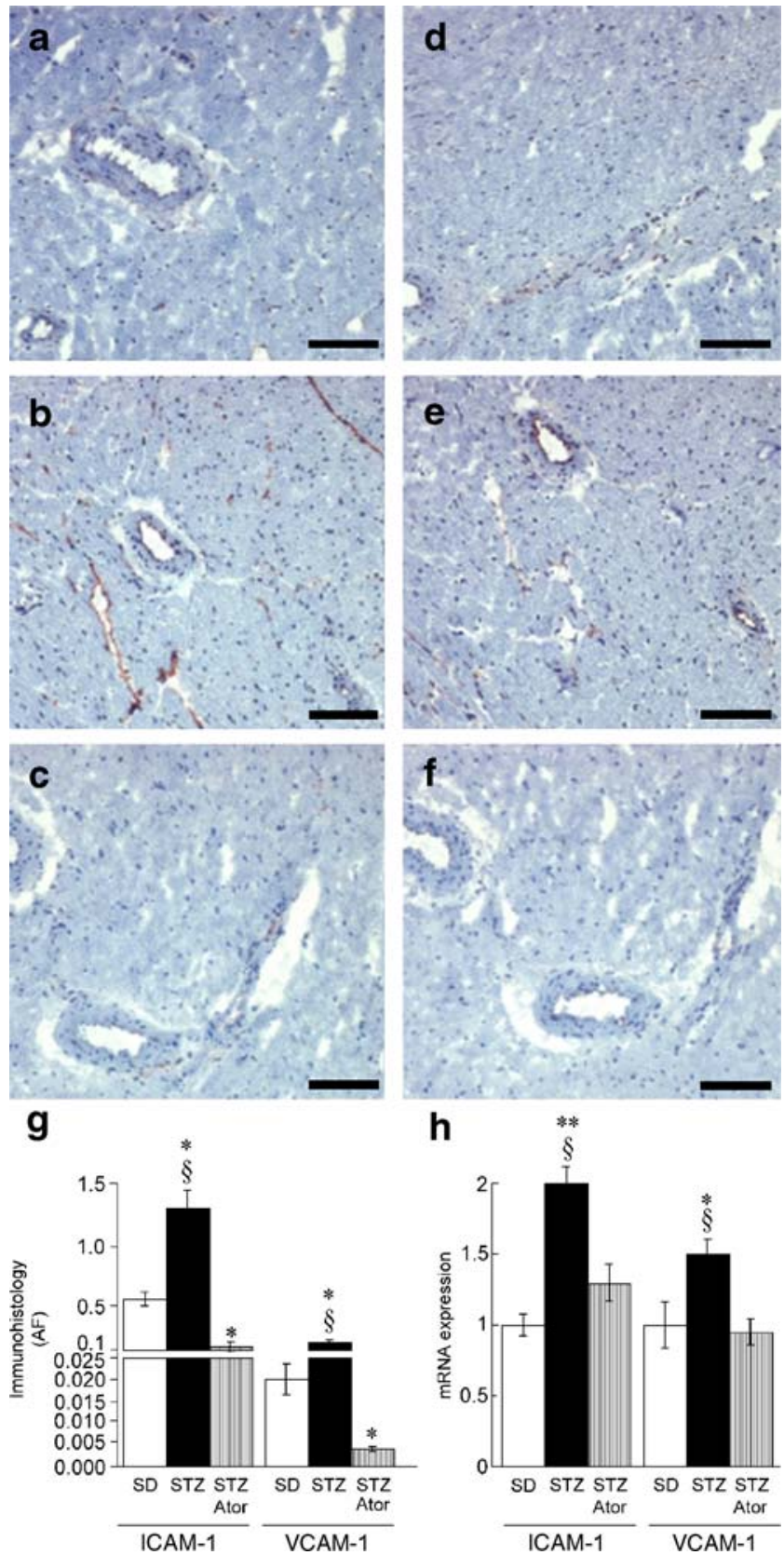

Fig. 2 Effect of atorvastatin treatment on cardiac CAMs expression in STZ-diabetic rat hearts. Immunohistochemistry of ICAM-1 (original magnification: $\times 100)$ in (a) a non-diabetic SD rat heart, (b) an STZdiabetic rat heart and (c) an atorvastatin-treated (Ator) STZ-diabetic rat heart. d-f Immunohistochemistry of VCAM-1 (original magnification: $\times 100$ ) in a non-diabetic SD rat heart, an STZ-diabetic rat heart and an atorvastatin-treated STZ-diabetic rat heart, respectively. Bar: $100 \mu \mathrm{m}$. Bar graph representing (g) quantification of ICAM-1 and VCAM-1 by immunohistochemistry ( $A F$, area fraction; $y$-axis break between 0.025 and 0.1 ) and (h) Icam-1 and Vcam-1 mRNA expression. mRNA of each gene was measured by real-time PCR and normalised to ribosomal $L 32$ mRNA expression. mRNA data are expressed as fold change from the level in SD control. Single asterisks $p<0.05$ and double asterisks $p<0.01 \mathrm{vs} \mathrm{SD,} \mathrm{section} \mathrm{marks} p<0.05$ vs $\mathrm{STZ}+$ atorvastatin 
suppressed the diabetes-induced infiltration of CD11a/LFA1+, CD11b/MAC1+ and CD18+ leucocytes to basal levels present in non-diabetic control rats (Fig. 3a) and reduced macrophage residence 2.8-fold $(p<0.05)$ (Fig. 3a). In addition, atorvastatin resulted in a 3.8-fold $(p<0.05)$ and 1.6-fold $(p<0.05)$ reduction of cardiac TNF- $\alpha$ - and IL- $1 \beta$-positive stainings in STZ rats, whereas in non-diabetic rats atorvastatin treatment did not affect cardiac cytokine levels (Fig. 3b).

Effect of atorvastatin treatment on RHOA-and RAC1GTPase activity in the diabetic rat heart To investigate the cholesterol-independent effects of atorvastatin, we analysed the activation of the small GTP-binding proteins, RAC1 and RHOA [11], which have both been suggested to be mediators of inflammation [23]. RAC1 and RHOAGTPase activity was 1.7 -fold $(p<0.05)$ and 1.6-fold $(p<0.05)$ upregulated in diabetic compared with non-diabetic control LVs, respectively. Atorvastatin treatment normalised RAC1and RHOA-GTPase activities to levels not significantly different from non-diabetic controls (Fig. 4a,b). Under nondiabetic conditions, atorvastatin further reduced RAC1- and RHOA-GTPase activity by 1.9 -fold $(p<0.05)$ and 1.6 -fold $(p<0.05)$, respectively.

Effect of atorvastatin treatment on p38 MAPK phosphorylation and lipid peroxide levels in the diabetic heart We further investigated the activation/phosphorylation state of MAPK p38, which is known to be induced by high glucose levels and to be involved in inflammation. We found that atorvastatin treatment in STZ diabetic rats resulted in a 3.2fold $(p<0.0005)$ decrease in diabetes-induced phosphorylation of p38 MAPK (Fig. 5a). Moreover, levels of the lipid peroxide malondialdehyde, a marker of oxidative stress, were reduced 1.2-fold $(p<0.05)$ in the diabetic heart after atorvastatin treatment (Fig. $5 b)$.

Effect of atorvastatin treatment on eNOS protein production in the diabetic rat heart Since RHOA activity is known to be associated with decreased levels of eNOS [12, 13], which partly due to its anti-inflammatory properties is an important marker of endothelial function, we investigated eNOS levels. In agreement with the reduction in RhoA activity found in atorvastatin-treated STZ rats, eNOS production was 1.3-fold $(p<0.05)$ higher in atorvastatin-treated compared with untreated STZ rats and was normalised to the expression levels found in non-diabetic controls (Fig. 6).

\section{Discussion}

The key finding of this study is that treatment with atorvastatin at a dose too low to decrease LDL-cholesterol levels reduces intramyocardial inflammation and myocardial fibrosis, resulting in a significantly improved LV function in a model of experimental type 1 diabetic cardiomyopathy.

Effect of atorvastatin on lipid profile The STZ-lipid profile shows moderately increased LDL-cholesterol, increased triacylglycerol and increased HDL-cholesterol [24, 25]. STZ-induced diabetes is characterised by a specific cholesterol metabolism [26], namely low cholesterol synthesis and high cholesterol absorption, mainly due to polyphagia and to a lesser extent to increased fractional absorption. Whereas statins are well known to reduce LDL-cholesterol and triacylglycerol and also to increase HDL-cholesterol [27],
Fig. 3 Effect of atorvastatin treatment (Ator) on $\beta_{2}$-leucocyteintegrins $^{+}$, macrophage residence and cytokine production in STZ-diabetic rat hearts. The graph shows quantification of infiltrates per $\mathrm{mm}^{2}$ of (a) CD11a, CD11b, CD18 and ED1/CD68 ( $y$-axis break between 5 and 10) and (b) TNF- $\alpha$ and IL-1 $\beta$ by immunohistochemistry. Sharp signs $p<0.05$ vs SD, section marks $p<0.05$ vs STZ +

atorvastatin, asterisks $p<0.05$ vs $\mathrm{SD}+$ atorvastatin
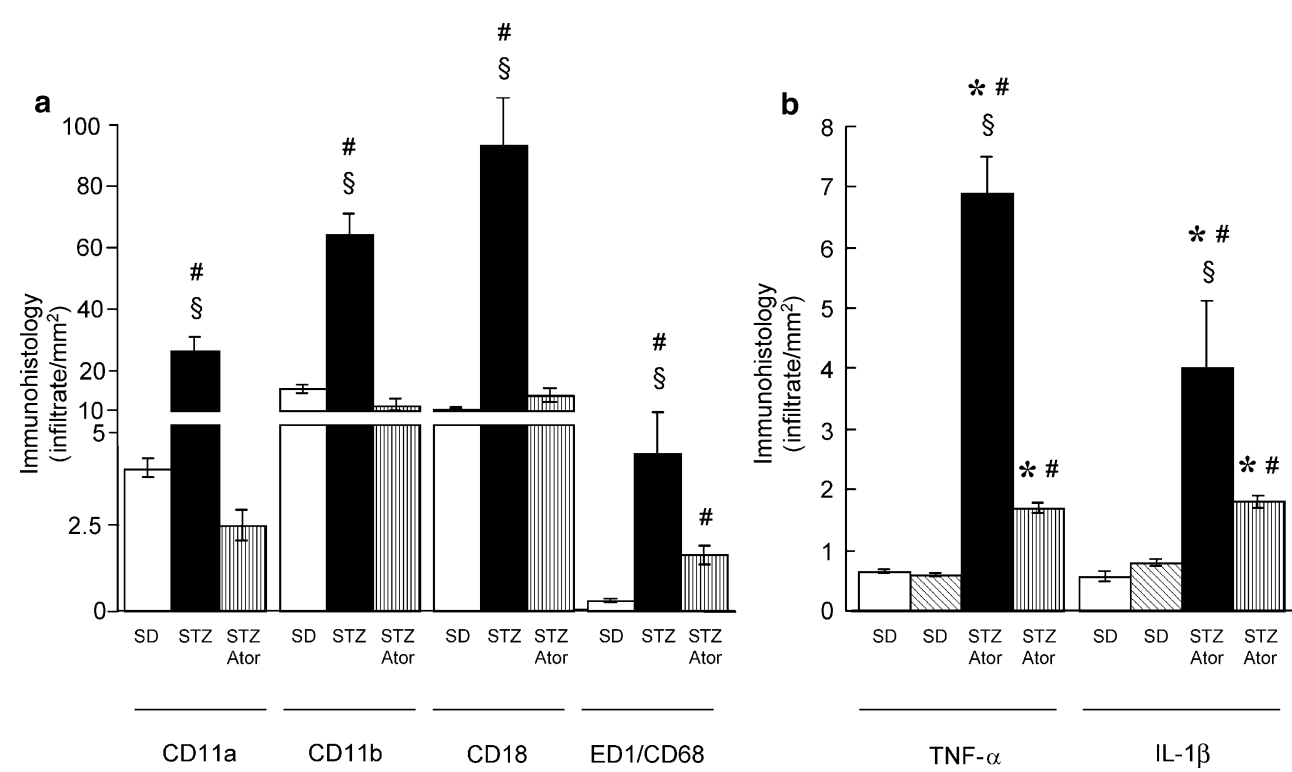
Fig. 4 Effect of atorvastatin treatment (Ator) on RAC1and RHOA-GTPase activity in STZ-diabetic rat hearts. a RAC1- and b RHOA-GTPase activity determined by pull-down assays. $n=6$ per group; asterisks $p<0.05$ vs $\mathrm{SD}$ a

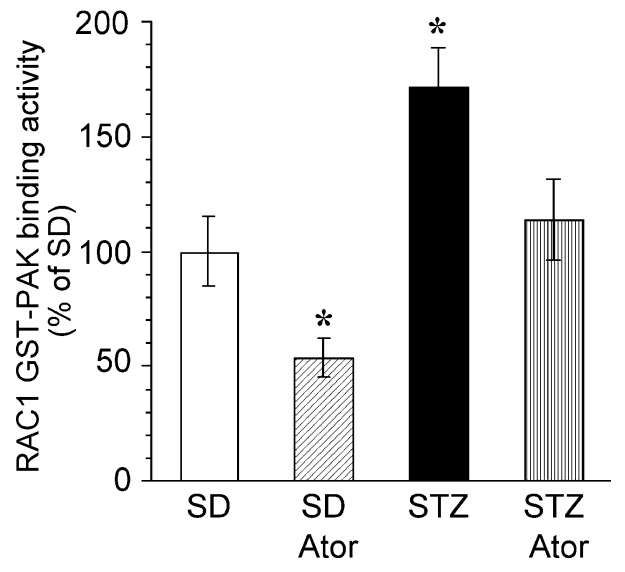

b

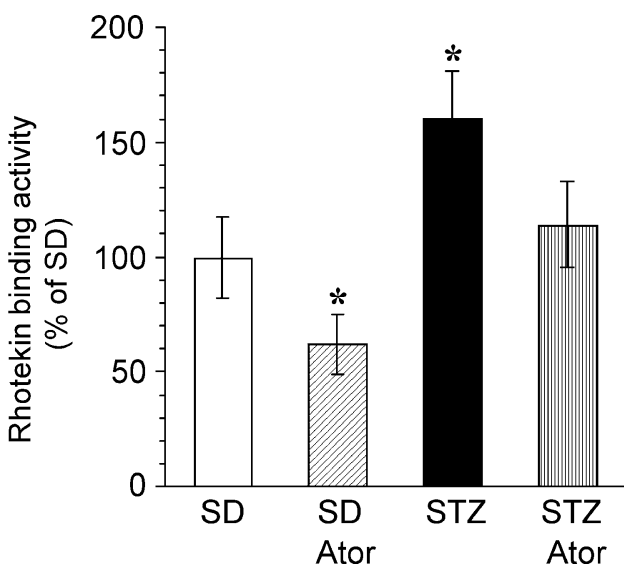

atorvastatin in STZ rats at the dose used here $(50 \mathrm{mg} / \mathrm{kg}$ daily, orally) did not decrease LDL-cholesterol and triacylglycerol or increase HDL-cholesterol. We hypothesise that the specific cholesterol metabolism in STZ-induced diabetes underlies this inhibited effect of atorvastatin treatment on the lipid profile. A potential explanation for the unaltered LDL-cholesterol levels after atorvastatin treatment is that an excess of exogenous cholesterol due to polyphagia desensitises the reaction to intracellular cholesterol depletion [28] induced by HMG CoA reductase inhibitors, which normally leads to increased LDL receptor production and increased LDL catabolism.

Other animal models such as spontaneous hypertensive rats [29] and young and only moderate diabetic rats [30] differ in this regard and have to be carefully and individually investigated. In our model of severe diabetes mellitus the atorvastatin dosage used did not significantly affect the lipid profile, allowing us to further investigate in vivo the pleiotropic effects of atorvastatin and its impact on the development of diabetic cardiomyopathy.

Effect of atorvastatin on LV function The STZ-model using $70 \mathrm{mg} / \mathrm{kg}$ i.p. is a well established model for the study of diabetic cardiomyopathy [31]. Insulin supplementation in animals injected with this dose of STZ leads to an improvement of LV function, indicating the role of hyperglycaemia in cardiac dysfunction [32]. Diabetic rats in our study already displayed severe LV dysfunction 47 days

Fig. 5 Effect of atorvastatin treatment (Ator) on p38 MAPK phosphorylation and lipid peroxide levels in STZ-diabetic rat hearts. a Representative western blots of phospho and total p38 MAPK, with bar graph (b) depicting the ratio of the band intensity of phospho:total p38 MAPK as $\%$ of SD set to $100 \% . n=4$ per group; asterisks $p<0.05$ vs $\mathrm{SD}$, sharp signs $p<0.01$ vs STZ + atorvastatin. c Malondialdehyde $(M D A)$ concentration (conc.)/protein concentration represented as \% of SD. $n=4-6$ per group. Asterisks $p<0.05$ vs SD, section marks $p<$ $0.05 \mathrm{vs} \mathrm{STZ}+$ atorvastatin a SD STZ STZ+Ator

p-p38 p38
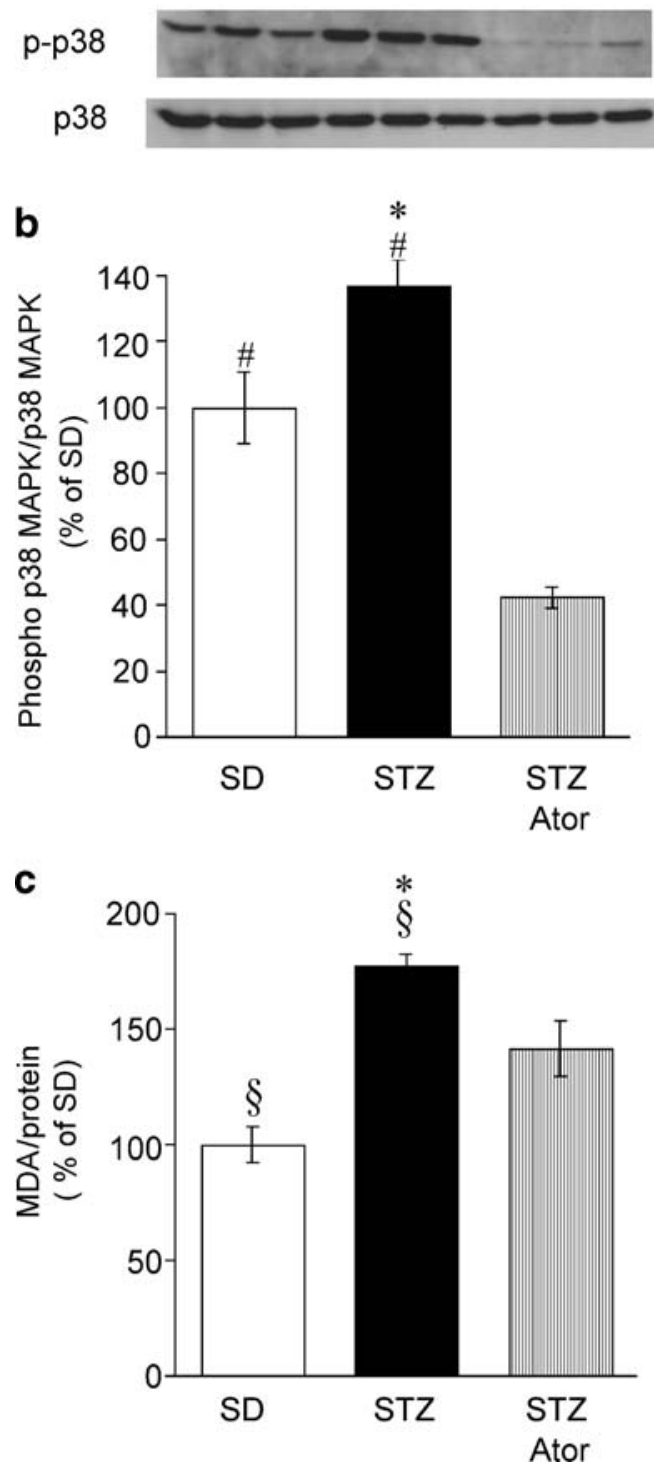

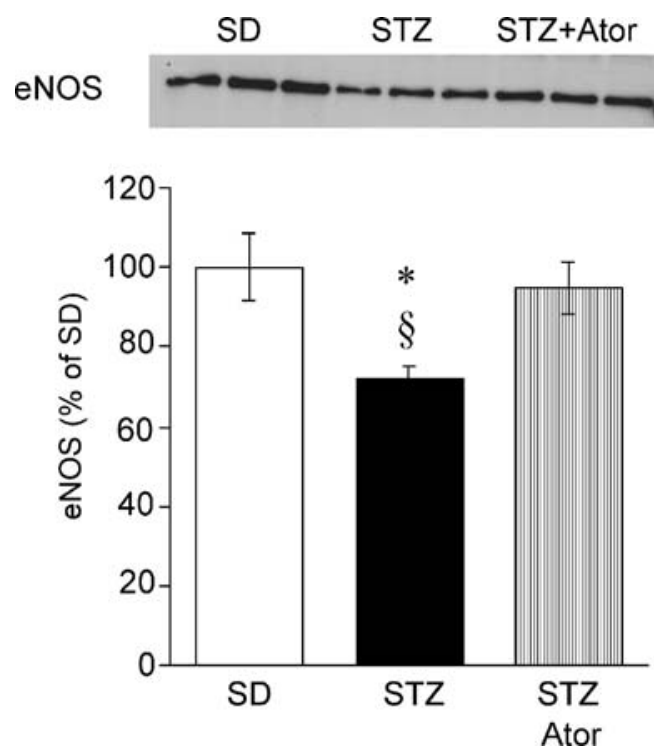

Fig. 6 Effect of atorvastatin treatment (Ator) on eNOS levels in STZdiabetic rat hearts. Representative western blots of total eNOS, with bar graph depicting the band intensity of total eNOS as \% of SD set to $100 \%$. $n=4$ per group. Asterisks $p<0.05$ vs SD, section marks $p<0.05$ vs STZ + atorvastatin

after STZ-injection, as indicated by impaired systolic and diastolic function [33], a finding which is in agreement with others [34-37]. Atorvastatin treatment in diabetic rats led to a significant improvement of systolic contraction and relaxation compared with untreated diabetic animals $\left(\mathrm{dP} / \mathrm{d} t_{\max }+95 \%, \mathrm{dP} / \mathrm{d} t_{\min }+64 \% ; p<0.05\right)$, whereas atorvastatin did not affect LV function in non-diabetic animals. The latter indicates that atorvastatin at a dose of $50 \mathrm{mg} / \mathrm{kg}$ daily in non-diabetic SD rats is not associated with any potential side effects, such as (cardiac) myopathy.

Whereas the cardioprotective effects of statins, independent of their hypolipidaemic effects, have already been demonstrated in several normocholesterolaemic models $[14,15,38]$, this is the first animal study demonstrating the pleiotropic effects of statins in a model characterised by increased LDL-cholesterol levels.

Effect of atorvastatin on myocardial fibrosis Changes in the extracellular matrix, and particularly in collagen composition, influence the passive mechanical properties of the myocardium [22]. Collagen I and III, which constitute $90 \%$ of cardiac collagen, are especially important for cardiac haemodynamics. Consistent with previous reports [19, 39], we found increased collagen I and III levels in the hearts of STZ-induced diabetic rats, correlating with LV dysfunction. In contrast, atorvastatin treatment blunted total cardiac collagen content and the levels of collagen I and III, in line with the improved LV function. Since myocardial fibrosis in diabetic cardiomyopathy is partly mediated by the upregulation of cytokines that have a pro-fibrotic action, including TNF- $\alpha$ and IL-1 $\beta$ [3], the reduction of myocar- dial inflammation may be one of the mechanisms involved in the anti-fibrotic effects of atorvastatin seen under STZdiabetic conditions.

Effect of atorvastatin on intramyocardial inflammation Many of the pleiotropic effects of statins are mediated by antagonism of isoprenoid-mediated activation of small GTP-binding proteins, including RAC1 and RHOA [11], which have previously been suggested to be mediators of inflammation [23, 40]. Both RAC1 [41] and RHOA GTPase [42] are involved in the regulation of the cytoskeleton network, which includes integrin-dependent leucocyte adhesion. RAC1 GTPase activity is essential for the activity of p38 MAPK [43], a crucial mediator of oxidative stress, a fact underscored by the cardiobeneficial effects of p38 inhibition under STZ-diabetic conditions [44]. However, this study is the first study illustrating: (1) increased activity of both RAC1 and RHOA in an experimental model of diabetic cardiomyopathy and (2) normalisation of RAC1 and RHOA activity, after atorvastatin treatment, to levels similar to those in non-diabetic controls. Interestingly, atorvastatin also reduced $\mathrm{RAC} 1$ and RHOA activity under non-diabetic conditions and confirmed that the chosen dose of atorvastatin was effective. However, LV function and cardiac cytokine levels were not changed, suggesting that under physiological conditions, a 1.9-fold and 1.6-fold reduction of the RAC1/RHOA pathway, respectively, is not sufficient to affect the cardiac phenotype or that this decrease is compensated by other signalling pathways.

In agreement with the diabetes-induced RAC1 activity, cardiac oxidative stress in diabetic rat hearts was increased compared with non-diabetic hearts. This was indicated by induced phosphorylation of p38 MAPK and increased cardiac lipid peroxide and malondialdehyde levels. In line with the increased activity of RHOA, which has been reported to decrease levels of eNOS [13], eNOS levels were reduced in diabetic rat hearts. In contrast, atorvastatin treatment resulted in normalisation of diabetes-induced RAC1 and RHOA levels in parallel with reduced p38 MAPK phosphorylation and lipid peroxide levels and increased levels of eNOS. In addition, CAM levels, leucocyte infiltrates and cytokine levels were reduced in atorvastatin-treated diabetic rat hearts. This suggests that atorvastatin, via reduction of RAC1 and RHOA activity associated with a reduction in cardiac oxidative stress and increased eNOS levels, reduces inflammation in diabetic cardiomyopathy. This hypothesis is supported by the finding that: (1) monocyte adhesion to vascular endothelium is reduced via statin-mediated downregulation of integrin adhesion molecules and inhibition of actin polymerisation via RHOA inactivation [40]; (2) atorvastatin treatment inhibits glucose-mediated neutrophil-endothelial 
cell adhesion through decreasing surface expression of endothelial adhesion molecules by stimulating the production of endothelial nitric oxide [45]; and (3) RHOA kinase inhibitors lead to increased eNOS levels and reduction of oxidative stress [13].

In conclusion, this study provides evidence that atorvastatin, independently of its LDL-cholesterol-lowering capacity, improves LV function via down-regulation of RAC1 and RHOA activity and reduction of inflammation and fibrosis.

Study limitations Since investigation of the development of LV dysfunction via human cardiac biopsies of type 1 diabetic patients is not possible, the well-established experimental STZ-model is often used to study this condition. However, it should be taken into account that this insulinopenic model is associated with extremely high blood glucose levels, which are not encountered in humans, as well as with dehydration, which accounts in part for the cardiac functional abnormalities. In addition, the differences between rodent and human lipoprotein metabolism make a direct translation of our findings, with the dose of atorvastatin used in this study, to humans impossible. Therefore, further studies are necessary to prove whether our results can be translated to humans with type 1 or type 2 diabetes mellitus.

Acknowledgements This study was supported by the Deutsche Forschungsgemeinschaft (GRK: 865).

Duality of interest The authors declare that there is no duality of interest associated with this manuscript.

\section{References}

1. Kannel WB, Hjortland M, Castelli WP (1974) Role of diabetes in congestive heart failure: the Framingham study. Am J Cardiol 34:29-34

2. Zarich SW, Nesto RW (1989) Diabetic cardiomyopathy. Am Heart J 118:1000-1012

3. Tschope C, Walther T, Escher F et al (2005) Transgenic activation of the kallikrein-kinin system inhibits intramyocardial inflammation, endothelial dysfunction and oxidative stress in experimental diabetic cardiomyopathy. FASEB J 19:2057-2059

4. Asbun J, Villarreal FJ (2006) The pathogenesis of myocardial fibrosis in the setting of diabetic cardiomyopathy. J Am Coll Cardiol 47:693-700

5. Lamarche B, Lemieux I, Despres JP (1999) The small, dense LDL phenotype and the risk of coronary heart disease: epidemiology, pathophysiology and therapeutic aspects. Diabetes Metab 25:199-211

6. King GL, Wakasaki H (1999) Theoretical mechanisms by which hyperglycemia and insulin resistance could cause cardiovascular diseases in diabetes. Diabetes Care 22(Suppl 3):C31-C37

7. Maytin M, Leopold J, Loscalzo J (1999) Oxidant stress in the vasculature. Curr Atheroscler Rep 1:156-164
8. Holvoet P, Collen D (1994) Oxidized lipoproteins in atherosclerosis and thrombosis. FASEB J 8:1279-1284

9. Sacks FM, Pfeffer MA, Moye LA et al (1996) The effect of pravastatin on coronary events after myocardial infarction in patients with average cholesterol levels. Cholesterol and Recurrent Events Trial investigators. N Engl J Med 335:1001-1009

10. Takemoto M, Liao JK (2001) Pleiotropic effects of 3-hydroxy-3methylglutaryl coenzyme a reductase inhibitors. Arterioscler Thromb Vasc Biol 21:1712-1719

11. Goldstein JL, Brown MS (1990) Regulation of the mevalonate pathway. Nature 343:425-430

12. Wolfrum S, Dendorfer A, Rikitake Y et al (2004) Inhibition of Rho-kinase leads to rapid activation of phosphatidylinositol 3kinase/protein kinase Akt and cardiovascular protection. Arterioscler Thromb Vasc Biol 24:1842-1847

13. Mita S, Kobayashi N, Yoshida K, Nakano S, Matsuoka H (2005) Cardioprotective mechanisms of Rho-kinase inhibition associated with eNOS and oxidative stress-LOX-1 pathway in Dahl saltsensitive hypertensive rats. J Hypertens 23:87-96

14. Lee TM, Lin MS, Chou TF, Tsai CH, Chang NC (2005) Effect of pravastatin on development of left ventricular hypertrophy in spontaneously hypertensive rats. Am J Physiol Heart Circ Physiol 289:H220-H227

15. Hayashidani S, Tsutsui H, Shiomi T et al (2002) Fluvastatin, a 3hydroxy-3-methylglutaryl coenzyme a reductase inhibitor, attenuates left ventricular remodeling and failure after experimental myocardial infarction. Circulation 105:868-873

16. Tschope C, Reinecke A, Seidl U et al (1999) Functional, biochemical, and molecular investigations of renal kallikreinkinin system in diabetic rats. Am J Physiol 277:H2333-H2340

17. Tschope C, Heringer-Walther S, Koch M et al (2000) Myocardial bradykinin B2-receptor expression at different time points after induction of myocardial infarction. J Hypertens 18:223-238

18. Pauschinger M, Knopf D, Petschauer S et al (1999) Dilated cardiomyopathy is associated with significant changes in collagen type I/III ratio. Circulation 99:2750-2756

19. Tschope C, Walther T, Koniger J et al (2004) Prevention of cardiac fibrosis and left ventricular dysfunction in diabetic cardiomyopathy in rats by transgenic expression of the human tissue kallikrein gene. FASEB J 18:828-835

20. Sander EE, van Delft S, ten Klooster JP et al (1998) Matrixdependent Tiam1/Rac signaling in epithelial cells promotes either cell-cell adhesion or cell migration and is regulated by phosphatidylinositol 3-kinase. J Cell Biol 143:1385-1398

21. Reid T, Furuyashiki T, Ishizaki T et al (1996) Rhotekin, a new putative target for Rho bearing homology to a serine/threonine kinase, PKN, and rhophilin in the rho-binding domain. J Biol Chem 271:13556-13560

22. Spinale FG (2002) Matrix metalloproteinases: regulation and dysregulation in the failing heart. Circ Res 90:520-530

23. Wassmann S, Laufs U, Baumer AT et al (2001) Inhibition of geranylgeranylation reduces angiotensin II-mediated free radical production in vascular smooth muscle cells: involvement of angiotensin AT1 receptor expression and Rac1 GTPase. Mol Pharmacol 59:646-654

24. Bar-On H, Roheim PS, Eder HA (1976) Serum lipoproteins and apolipoproteins in rats with streptozotocin-induced diabetes. J Clin Invest 57:714-721

25. Bar-On H, Eisenberg S (1978) The metabolic fate of high density lipoprotein (HDL) in the diabetic rat. Diabetologia 14:65-69

26. Young NL, Lopez DR, McNamara DJ (1988) Contributions of absorbed dietary cholesterol and cholesterol synthesized in small intestine to hypercholesterolemia in diabetic rats. Diabetes 37 : $1151-1156$

27. Schaefer JR, Schweer H, Ikewaki K et al (1999) Metabolic basis of high density lipoproteins and apolipoprotein A-I increase by 
HMG-CoA reductase inhibition in healthy subjects and a patient with coronary artery disease. Atherosclerosis 144:177-184

28. Rawson RB (2003) The SREBP pathway-insights from Insigs and insects. Nat Rev Mol Cell Biol 4:631-640

29. Wassmann S, Laufs U, Baumer AT et al (2001) HMG-CoA reductase inhibitors improve endothelial dysfunction in normocholesterolemic hypertension via reduced production of reactive oxygen species. Hypertension 37:1450-1457

30. Mahfouz MM, Kummerow FA (2005) Atorvastatin reduces the plasma lipids and oxidative stress but did not reverse the inhibition of prostacyclin generation by aortas in streptozotocin diabetic rats. Prostaglandins Other Lipid Mediat 76:59-73

31. Tomlinson KC, Gardiner SM, Hebden RA, Bennett T (1992) Functional consequences of streptozotocin-induced diabetes mellitus, with particular reference to the cardiovascular system. Pharmacol Rev 44:103-150

32. Schaible TF, Malhotra A, Bauman WA, Scheuer J (1983) Left ventricular function after chronic insulin treatment in diabetic and normal rats. J Mol Cell Cardiol 15:445-458

33. Litwin SE, Raya TE, Anderson PG, Daugherty S, Goldman S (1990) Abnormal cardiac function in the streptozotocin-diabetic rat. Changes in active and passive properties of the left ventricle. $J$ Clin Invest 86:481-488

34. Koch M, Wendorf M, Dendorfer A et al (2003) Cardiac kinin level in experimental diabetes mellitus: role of kininases. Am J Physiol Heart Circ Physiol 285:H418-H423

35. Bidasee KR, Zhang Y, Shao CH et al (2004) Diabetes increases formation of advanced glycation end products on Sarco(endo) plasmic reticulum $\mathrm{Ca}^{2+}$-ATPase. Diabetes 53:463-473

36. Vasanji Z, Dhalla NS, Netticadan T (2004) Increased inhibition of SERCA2 by phospholamban in the type I diabetic heart. Mol Cell Biochem 261:245-249
37. Montanari D, Yin H, Dobrzynski E et al (2005) Kallikrein gene delivery improves serum glucose and lipid profiles and cardiac function in streptozotocin-induced diabetic rats. Diabetes 54:1573-1580

38. Lefer AM, Campbell B, Shin YK, Scalia R, Hayward R, Lefer DJ (1999) Simvastatin preserves the ischemic-reperfused myocardium in normocholesterolemic rat hearts. Circulation 100:178-184

39. Modrak J (1980) Collagen metabolism in the myocardium from streptozotocin-diabetic rats. Diabetes 29:547-550

40. Yoshida M, Sawada T, Ishii H et al (2001) Hmg-CoA reductase inhibitor modulates monocyte-endothelial cell interaction under physiological flow conditions in vitro: involvement of Rho GTPasedependent mechanism. Arterioscler Thromb Vasc Biol 21:1165-1171

41. Khayat ZA, Tong P, Yaworsky K, Bloch RJ, Klip A (2000) Insulin-induced actin filament remodeling colocalizes actin with phosphatidylinositol 3-kinase and GLUT4 in L6 myotubes. J Cell Sci 113(Pt 2):279-290

42. Wojciak-Stothard B, Williams L, Ridley AJ (1999) Monocyte adhesion and spreading on human endothelial cells is dependent on Rho-regulated receptor clustering. J Cell Biol 145:1293-1307

43. Uhlik MT, Abell AN, Johnson NL et al (2003) Rac-MEKK3MKK3 scaffolding for p38 MAPK activation during hyperosmotic shock. Nat Cell Biol 5:1104-1110

44. Westermann D, Rutschow S, Van Linthout S et al (2006) Inhibition of p38 mitogen-activated protein kinase attenuates left ventricular dysfunction by mediating pro-inflammatory cardiac cytokine levels in a mouse model of diabetes mellitus. Diabetologia 49:2507-2513

45. Omi H, Okayama N, Shimizu M et al (2003) Statins inhibit high glucose-mediated neutrophil-endothelial cell adhesion through decreasing surface expression of endothelial adhesion molecules by stimulating production of endothelial nitric oxide. Microvasc Res 65:118-124 